\title{
A Case of Post Varicella Recurrent Presternal Butterfly Keloid
}

\author{
Andre Vera ${ }^{1}$, Henry Weatherburn², Kamalendu Malaker ${ }^{3,4 *}$ \\ ${ }^{1}$ Radiotherapia Oncologica GURVE, Instituto Medico la Floresta, Caracus, Venezuela \\ ${ }^{2}$ Parkside Oncology Clinic, London, UK \\ ${ }^{3}$ ICM Department, Ross University School of Medicine, Portsmouth, Dominica \\ ${ }^{4}$ Princess Margaret Hospital, Roseau, Dominica \\ Email: avera@ radiotherapia.com.ve, henryweatherburn@cancer, ${ }^{*}$ kmalake@rossmed.edu.dm
}

Received March 23, 2013; revised April 17, 2013; accepted May 27, 2013

Copyright (C) 2013 Andre Vera et al. This is an open access article distributed under the Creative Commons Attribution License, which permits unrestricted use, distribution, and reproduction in any medium, provided the original work is properly cited.

\begin{abstract}
Presternal butterfly keloid management remains a clinical challenge. This case indicates, with hypo fractionated external beam electron therapy a significant symptomatic and cosmetic benefit may be achieved without any unacceptable acute, chronic or long term toxicity.
\end{abstract}

Keywords: Butterfly Presternal Keloid; Hypo Fractionated External Beam Radiotherapy

\section{Patient}

With 15 years prior history of varicella attack, a 31-year Hispanic Venezuelan healthy male, presented in January 2011. He had several satellite keloids surrounding the central major lesion, all being developed in a post varicella scar in the anterior chest wall. Since then he has been through three surgical excisions and on each occasion the lesion recurred with worsening size and symptoms. His last resection was carried out in June 2010. By the end of 2010 he recurred again with further worsening symptom and progressive lesion. He was not considered for further resection and was referred for radiotherapy.

He was seen in radiotherapy in January 2011. He presented with a large mid presternal butterfly keloid measuring $8.5 \mathrm{~cm} \times 4-6 \mathrm{~cm}$. Surrounded by 5 satellite keloids $0.5-0.75 \times 0.75-1.5 \mathrm{~cm}$. The lesions were pink, tender, hard nodular, fibrotic, criss-crossing with hard fibrotic bands especially in the main lesion. Height varied between 3 - $10 \mathrm{~mm}$. The total skin area that encompasses the main and satellite lesions is $9.5 \times 9.5 \mathrm{~cm}^{2}$. Aside his progressive symptoms of severe pain and itching not controlled by medications and topical applications; unsightly appearance restricting his social interaction and personal embarrassment has led to depression and voluntary isolation.

There was no other keloid or hypertrophic scar in hisbody, did not give any specific history of allergy or *Corresponding author. atopic condition. He was not diabetic, nor had any keloid formers in the family. He did not have any African or south east Asian genetic heritage as far he knows.

Keloids arising in varicella scar are particularly resistant to available treatments [1]. Presternal butterfly keloids respond poorly to conventional radiotherapy or other form of supportive treatments. Multiple surgical resections make the keloid size bigger more fibrotic and hypoxic [2]. Hence this particular lesion had all the risk factors against achieving any reasonable result from conventional radiotherapy or combinations of modalities [3, 4]. So it was decided to treat him with Hypo fractionated external beam radiotherapy [5] primarily for symptomatic relief and secondarily for cosmesis by achieving some degree of size reduction and flattening of the lesion/s. For measurement of reaction and response, clinical photographs were taken, before, during, after each fraction and frequently during follow up to 12 months.

\section{Radiotherapy Planning and Prescription}

Anticipated risk of treating presternal region with megavoltage radiotherapy is the radiation dosage to the heart and other mediastinal structures. Hence it was decided to check the dosimetry with CT scans for Radiotherapy dosimetry using appropriate Electron Beams. Electron beam was chosen to reduce medistinal radiation and achieve better cosmesis $[5,6]$. Cosmesis is a major concern in this patient. 
$6 \mathrm{MeV}$ Electron beam, using a $15 \times 15 \mathrm{~cm}^{2}$ field with lead cut out for an area of $11.5 \times 11.5 \mathrm{~cm}^{2}$ was prepared. Since the maximum height of the lesion is approximately $10 \mathrm{~mm}$ thick; a $0.5-\mathrm{mm}$ wax bolus was added on the surface of lesion (Figures 1(a) and (b)).

In absence of CT scan of the chest of this patient; in- stead of using a phantom, we chose to use a CT scan of a male of comparable age, ethnicity, BMI and chest dimensions etc., to work out the dosimetry. A CT scan of the chest both transverse and longitudinal section was used to verify the likely depth dose and dosage to the critical mediastinal structures (Figures 1(c) and (d)). Thus
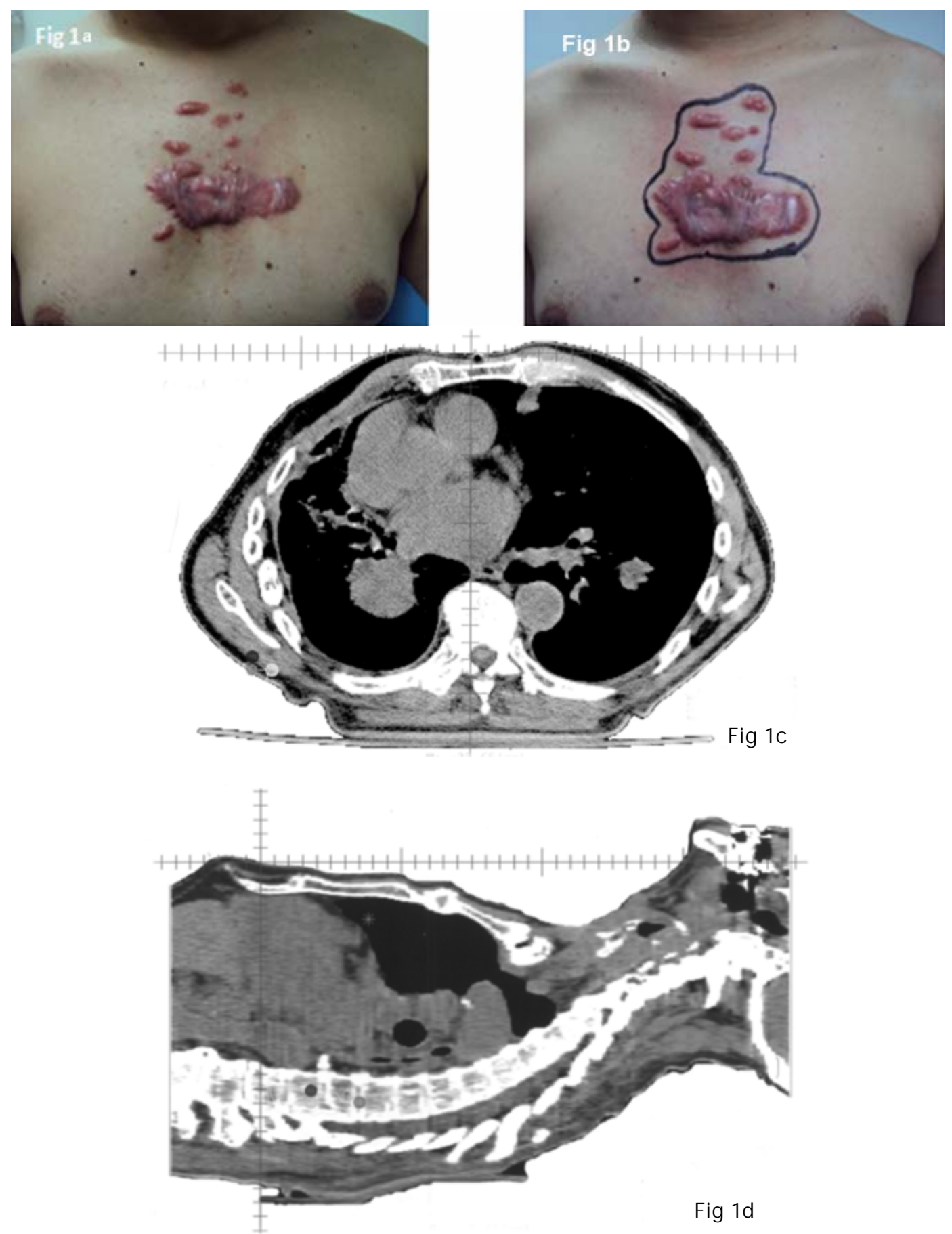

Figure 1. (a) Presented with presternal butterfly keloid with multiple satellite keloids on 8th February 2011; (b) Shows the outline of the entire area of the skin to be treated encompassing the primary butterfly keloid and its satellite lesions adding 2 $\mathrm{mm}$ to the outer border of all keloids [5]; (c) Depth dose in CT scan on Transverse section; (d) CT scan in sagittal section of the central sagittal axis of the lesion: cardiac and mediastinal structures. 
the detail of prescription and dosimetry are as follows:

- Total dose prescribed-3750 cGy in 5 weekly fractions at 750 cGy per fraction at 95\% depth dose, over 4 weeks.

- $6 \mathrm{MeV}$ electron beam on linear accelerator was given.

- A $15 \times 15 \mathrm{~cm}^{2}$ field was chosen to cover $11.5 \times 11.5$ $\mathrm{cm}^{2}$ fields with lead cut out.

- $0.5-\mathrm{cm}$ thick wax bolus was used on the surface of the lesion.

- Skin surface dose (under $0.5 \mathrm{~cm}$ bolus) $=89 \%$ i.e. 3340 cGy (3750 cGy at 100\%).

- Maximum dose at rear of the lesion (at $0.7 \mathrm{~cm}$ depth in the lesion, i.e. $1.2 \mathrm{~cm}$ from the surface of the bolus $=100 \%$, i.e. $3750 \mathrm{cGy}$.

- Dose at the rear of the lesion (at $1.0 \mathrm{~cm}$ depth, i.e. 1.5 $\mathrm{cm}$ from the surface of the bolus) $=96 \%$, i.e. 3600 cGy.

- Dose at the rear of the sternum (Approx. $1.75 \mathrm{~cm}$ as per the CT scan, i.e. $2.25 \mathrm{~cm}$ from the surface of the bolus) $=48 \%$, i.e. $1800 \mathrm{cGy}$.

- Dose at the anterior aspect of the heart (Approx. 2.5 $\mathrm{cm}$ as per the CT scan, i.e. $3 \mathrm{~cm}$ from the surface of the bolus) $=4 \%$ i.e. $150 \mathrm{cGy}$.

(We calculated the radiation dose, taking sternum to be of unit density.)

He was prescribed at 95\% level. So the Tumor dose was $3750 / 0.95=3950 \mathrm{cGy}$, at the rear of the lesion to 3600/0.95 = 3790 cGy.

The cardiac dose will range from 250 - 300 cGy over a period of 4 weeks or 28 days.

Depth dose to the mediastinum are displayed both in transverse and sagittal plane at the mid-level of the central lesion.

\section{Tolerance and Response to Hypo Fractionated Radiotherapy}

Patient tolerated the treatment well. Just after the first fraction, he experienced some alleviation of his symptom. Figure 2(a) (22.6.2011) shows skin reaction 1 week after first fraction, mild erythema without any desquamation or edema. Figure 2(b) (21.7.2011) shows extent of skin reaction on the last or the 5 th fraction i.e. 28 days after treatment started, significant bright erythema with coalescence of moist desquamation, especially on the main lesion on higher thickness of the lesion. There was no detectable keloid regression or flattening were noted, there was some dry desquamation noted (Gr3), No significant pain or discomfort from the reaction, reported by the patient Figure 2(c) (12.8.2011) shows extent of skin reaction 3 weeks after last fraction, persistent deep but fading erythema, areas of dry desquamation, less extensive, coalesced moist desquamation, but contracted area, patchy areas of keloid flattening was noted. There was no detectable edema or pain/tenderness noted (improving
Gr3). Figure 2(d) (31.7.2012) shows 12 months after treatment completed. All satellite keloids regressed by $80 \%$ of their height and areas to some extent. Main pre sternal butterfly lesion also regressed between 30\% and $50 \%$ of itsheight. Erythema remains as slight pigmentation, desquamated areas healed completely, the lesions are softer. Patient keloid symptoms had resolved on completion of his last fraction. He did not feel socially restricted or compromised, felt very happy to return to his social life and commitments.
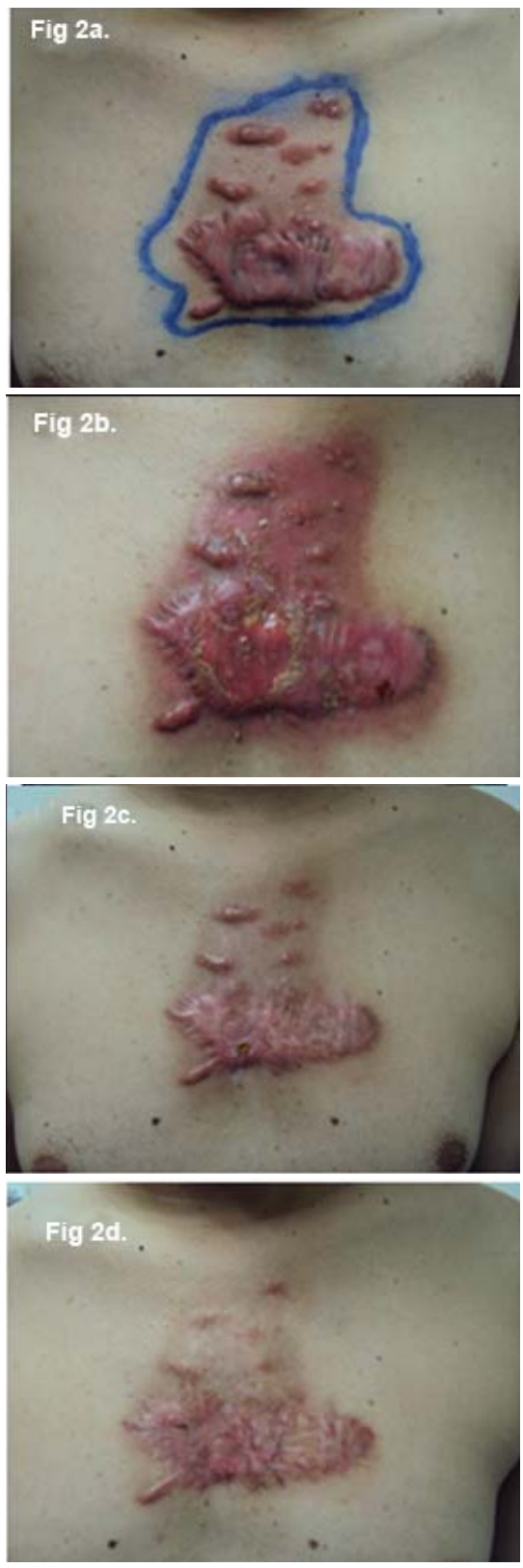

Figure 2. (a) (22.6.2011) shows skin reaction 1 week after first fraction; (b) (21.7.2011) shows extent of skin reaction on the last or the 5th fraction; (c) (12.8.2011) shows extent of skin reaction 3 weeks after last fraction; (d) (31.7.2012) shows 12 months after treatment completed. 


\section{Comments}

Presternal butterfly keloids are very difficult to treat. Symptomatic relief with standard external beam radiotherapy may be achieved to some extent, but reduction of keloid bulk happens rarely, specially of the presternal keloids, keloids arising in varicella scars [7] and one's had multiple surgical resections [2] tends to respond poorly to any form of treatment. Intra-lesional injections of medications, compression with or without silicone gel sheets, radiotherapy of any form, fares with very limited success $[3,4]$. This lesion is a clinical challenge having several poor prognostic indicators and little documented record reporting management of such a lesion. Using combinations of concurrent multi-modality treatment, Malaker et al. [8] in a series of 71 cases of recurrent or "difficult to treat keloids" reported some success. But in their series of 71 cases, 5 presternal butterfly keloids, none had multiple poor prognostic indicators like thiscase. They injected the entire lesion with combination of triamcinolone, dexamethasone and hyaluronidase, weekly for 6 - 8 weeks. Immediately after the injection the lesions were covered with silicone gel sheets and compressed with 1.5 - $2 \mathrm{~cm}$ thick hard bee wax blocks customized to the shape of the keloid, and compressed and retained with elastoplast bandage, for one week and repeat the procedure weekly [9-11]. It is cheap and suitable for, children, adolescence and women. But the intra lesional injections are painful and 6 - 8 weeks of elastoplast compression is some challenge to patients for their comfort and tolerance. No other specific reports available particularly were focusing on the management of presternal keloids. But general consensus is that these are very difficult lesions to treat or to achieve any meaningful palliation.

This particular case indicatesbutterfly presternal keloids may be treated with hypo fractionate delectron external beam therapy, to achieve meaningful symptomatic and cosmetic benefit. This patient will continue to improve from cosmetic point of view for next few years, with further regression and flattening of all the lesions.

\section{Acknowledgements}

I wish to thank Irus Toussaint for technical assistance and Ms. Kristen Campbell for editorial help and Ms. Joan Joseph for secretarial assistance.

\section{REFERENCES}

[1] N. Kluger, A. Mahe and B. Guillot, "Eruptive Keloids after Chickenpox,” Dermatology Reports, Vol. 3, No. 2, 2011, p. 35.

[2] A. Bayat, G. Arscott, W. E. R. Oliver and M. W. J. Ferguson, “'Aggressive Keloids”: A Severe Variant of Familial Keloid Scaring,” Journal of the Royal Society of Medicine, Vol. 96, No. 11, 2003, pp. 554-555.

[3] M. A. Daizi, N. A. Chowdri, S. K. Kaul and M. Khan, "Evaluation of Various Methods of Treating Keloids and Hyper Tropic Scars,” British Journal of Plastic Surgery, Vol. 45, No. 5, 1992, pp. 374-379.

[4] O. Rei, "Most Current Algorithms for Treatment and Prevention of Hypertrophic Scars and Keloids," Plastic and Reconstructive Surgery, Vol. 125, No. 2, 2010, pp. 557-568.

[5] K. Malaker, V. Vijayragavan and I. Hodson, "Retrospective Analysis of Treatment of Unresectable Keloids with Primary Radiation over 25 Years," Clinical Oncology, Vol. 16, No. 4, 2004, pp. 290-298.

[6] O. Rei, M. Kioshi, et al., "Postoperative Electron Beam Irradiation Therapy for Keloids," Plastic and Reconstructive Surgery, Vol. 111, No. 2, 2003, p. 547.

[7] N. Sceinfield and S. R. Cohen, "Varicella Causes Skin Pits and Keloids-More Reasons for Varicella Vaccine," Pediatrics, Vol. 106, No. 1, 2000, p. 160.

[8] K. Malaker, M. Zaidi, F. Ridda and T. Al Yafi, "Concomitant Multimodality Treatment of Keloids (CMTK) Unmanageable by Conventional Post-Operative Radiotherapy," 9th Congress of the Pan Arab Association of Burns and Plastic Surgery, Bahrain, 11-13 April 2006.

[9] K. Malaker, M. Zaidi, F. Ridda and T. Al Yafi, "Update on Concomitant Multimodality Treatment of Keloids (CMTK) Unmanageable by Conventional Postoperative Radiotherapy," 17th Annual Meeting of the European Association of Plastic Surgeon (EUROPAS), Istanbul, 25-27 May 2006.

[10] K. Malaker, M. Zaidi and F. Ridda, “'Tripoli Protocol’: A Developing Nation's Challenge in Managing Some Difficult Keloids,” International Scar Meeting, Tokyo, 30 November-1 December 2010.

[11] K. Malaker, M. Zaidi, M. R. Franka and T. Al Yafi, “Concurrent Multi-Modality Treatment of Keloids (CMTK) Not Manageable by Conventional Postoperative Radiotherapy," International Journal of Clinical Medicine, Vol. 4, No. 5, 2013, pp. 273-281. 